\title{
Pacific
}

Journal of

Mathematics

\section{DISTORTION OF BOUNDARY SETS UNDER INNER FUNCTIONS. II}

Jose Luis Fernandez Perez, Domingo Pestana And José Rodríguez 


\section{DISTORTION OF BOUNDARY SETS UNDER INNER FUNCTIONS (II)}

José L. Fernández, Domingo Pestana and José M. Rodríguez

We present a study of the metric transformation properties of inner functions of several complex variables. Along the way we obtain fractional dimensional ergodic properties of classical inner functions.

\section{Introduction.}

An inner function is a bounded holomorphic function from the unit ball $\mathbb{B}_{n}$ of $\mathbb{C}^{n}$ into the unit disk $\Delta$ of the complex plane such that the radial boundary values have modulus 1 almost everywhere. If $E$ is a non empty Borel subset of $\partial \Delta$, we denote by $f^{-1}(E)$ the following subset of the unit sphere $\mathbb{S}_{n}$ of $\mathbb{C}^{n}$

$$
f^{-1}(E)=\left\{\xi \in \mathbb{S}_{n}: \lim _{r \rightarrow 1} f(r \xi) \text { exist and belongs to } E\right\}
$$

The classical lemma of Löwner, see e.g. [R, p. 405], asserts that inner functions $f$, with $f(0)=0$, are measure preserving transformations when viewed as mappings from $\mathbb{S}_{n}$ to $\partial \Delta$, i.e. if $E$ is a Borel subset of $\partial \Delta$ then $\left|f^{-1}(E)\right|=|E|$, where in each case $|\cdot|$ means the corresponding normalized Lebesgue measure.

In this paper we extend this result to fractional dimensions as follows:

Theorem 1. If $f$ is inner in the unit disk $\Delta, f(0)=0$, and $E$ is a Borel subset of $\partial \Delta$, we have:

$$
\operatorname{cap}_{\alpha}\left(f^{-1}(E)\right) \geq \operatorname{cap}_{\alpha}(E), \quad 0 \leq \alpha<1 .
$$

Moreover, if $E$ is any Borel subset of $\partial \Delta$ with $\operatorname{cap}_{\alpha}(E)>0$, equality holds. if and only if either $f$ is a rotation or $\operatorname{cap}_{\alpha}(E)=\operatorname{cap}_{\alpha}(\partial \Delta)$.

Moreover, it is well known, see [N], that if $f$ is not a rotation then $f$ is ergodic, i.e., there are no nontrivial sets $A$, with $f^{-1}(A)=A$ except for a set of Lebesgue measure zero. This also has a fractional dimensional parallel. 
Corollary. With the hypotheses of Theorem 1 , if $f$ is not a rotation and if the symmetric difference between $E$ and $f^{-1}(E)$ has zero $\alpha$-capacity, then either $\operatorname{cap}_{\alpha}(E)=0$ or $\operatorname{cap}_{\alpha}(E)=\operatorname{cap}_{\alpha}(\partial \Delta)$.

Theorem 2. If $f$ is inner in the unit ball of $\mathbb{C}^{n}, f(0)=0$, and $E$ is a Borel subset of $\partial \Delta$, we have:

$$
\operatorname{cap}_{2 n-2+\alpha}\left(f^{-1}(E)\right) \geq K(n, \alpha)^{-1} \operatorname{cap}_{\alpha}(E), \quad 0<\alpha<1,
$$

and

$$
\frac{1}{\operatorname{cap}_{2 n-2}\left(f^{-1}(E)\right)} \leq 1+(2 n-2) \log \frac{1}{\operatorname{cap}_{0}(E)}, \quad(n>1)
$$

Corollary. In particular, for any inner function $f$, we have that

$$
\operatorname{Dim}\left(f^{-1}(E)\right) \geq 2 n-2+\operatorname{Dim}(E)
$$

where Dim denotes Hausdorff dimension.

Here $\operatorname{cap}_{\alpha}$ and cap $_{0}$ denote, respectively, $\alpha$-dimensional Riesz capacity and logarithmic capacity. We refer to $[\mathbf{C}],[\mathbf{K S}]$ and $[\mathbf{L}]$ for definitions and basic background on capacity.

For background and some applications of these results we refer to $[\mathbf{F P}]$ where it is shown that Theorem 1 holds with some constants depending on $\alpha$.

The outline of this paper is as follows: In Section 2 we obtain an integral expression for the $\alpha$-energy that is used in Section 3, where Theorems 1 and 2 are proved. Section 4 contains some further results for the case $n=1$. In Section 5, we prove an analogous distortion theorem, with Hausdorff measures replacing capacities. Section 6 discusses an open question and some partial results concerning distortion of subsets of the disc.

We would like to thank José Galé and Francisco Ruíz-Blasco for some helpful conversations concerning the energy functional. Also, we would like to thank David Hamilton for suggesting that the right constant in Theorem 1 is 1 (see $[\mathbf{H}]$ ), and the referee for some valuable comments.

\section{An integral expression for the $\alpha$-energy.}

In this section we obtain an expression of the $\alpha$-energy of a signed measure $\mu$ in $\Sigma_{N-1}$ (the unit sphere of $\mathbb{R}^{N}$ ) as an $L^{2}$-norm of its Poisson extension. This approach is due to Beurling $[\mathbf{B}]$. 
If $\mu$ is a signed measure on $\Sigma_{N-1}$, and $0 \leq \alpha<N-1$, then the $\alpha$-energy $I_{\alpha}(\mu)$ of $\mu$ is defined as

$$
I_{\alpha}(\mu)=\iint_{\Sigma_{N-1} \times \Sigma_{N-1}} \Phi_{\alpha}(|x-y|) d \mu(x) d \mu(y),
$$

where

$$
\Phi_{\alpha}(t)= \begin{cases}\log \frac{1}{t}, & \text { if } \alpha=0, \\ \frac{1}{t^{\alpha}}, & \text { if } 0<\alpha<N-1 .\end{cases}
$$

Recall that if $E$ is a closed subset of $\Sigma_{N-1}$, then

$\left(\operatorname{cap}_{\alpha}(E)\right)^{-1}=\inf \left\{I_{\alpha}(\mu): \mu\right.$ a probability measure supported on $\left.E\right\}$,

for $0<\alpha<N-1$,

$\log \frac{1}{\operatorname{cap}_{0}(E)}=\inf \left\{I_{0}(\mu): \mu\right.$ a probability measure supported on $\left.E\right\}$,

and that the infimum is attained by a unique probability measure $\mu_{e}$ which is called the equilibrium distribution of $E$.

If $E$ is any Borel subset of $\Sigma_{N-1}$, then the $\alpha$-capacity of $E$ is defined as

$$
\operatorname{cap}_{\alpha}(E)=\sup \left\{\operatorname{cap}_{\alpha}(K): K \subset E, K \text { compact }\right\} \text {. }
$$

We recall Choquet's theorem that all Borel sets are capacitables, i.e.

$$
\operatorname{cap}_{\alpha}(E)=\inf \left\{\operatorname{cap}_{\alpha}(O): E \subset O, O \text { open }\right\} .
$$

As we shall remark later on, for a general Borel set $E$ of $\Sigma_{N-1}$, one has

$$
\frac{1}{\operatorname{cap}_{\alpha}(E)}=\inf \left\{I_{\alpha}(\mu): \mu \text { a probability measure, } \mu(E)=1\right\}
$$

and analogously for the logarithmic capacity.

We first need to obtain the expansion of the integral kernel $\Phi_{\alpha}$ in terms of the spherical harmonics. We refer to [SW, Chap. IV] for details about spherical harmonics; we shall follow its notations.

Let $\mathcal{H}_{k}$ be the real vector space of the spherical harmonics of degree $k$ in $\mathbb{R}^{N}(N>1)$. If $a_{k}$ is the dimension of $\mathcal{H}_{k}$, we have

$$
a_{0}=1, \quad a_{1}=N, \quad a_{k}=\frac{N+2 k-2}{k}\left(\begin{array}{c}
N+k-3 \\
k-1
\end{array}\right) .
$$


If $\Sigma_{N-1}$ denotes the unit sphere of $\mathbb{R}^{N}$, the space $L^{2}\left(\Sigma_{N-1}, d \xi\right)$ can be decomposed as

$$
L^{2}\left(\Sigma_{N-1}, d \xi\right)=\bigoplus_{k=0}^{\infty} \mathcal{H}_{k}
$$

where $d \xi$ is the usual Lebesgue measure (not normalized).

If $\xi, \eta$ belongs to $\Sigma_{N-1}, Z_{\eta}^{k}(\xi)$ will denote the zonal harmonic of degree $k$ with pole $\eta$, and if $\left\{Y_{1}^{k}, \ldots, Y_{a_{k}}^{k}\right\}$ is any orthonormal basis of $\mathcal{H}_{k}$, we have

$$
Z_{\eta}^{k}(\xi)=\sum_{m=1}^{a_{k}} Y_{m}^{k}(\xi) Y_{m}^{k}(\eta)=Z_{\xi}^{k}(\eta)
$$

The zonal harmonics can be expressed in terms of the ultraspherical (or Gegenbauer) polynomials $P_{k}^{\lambda}$ which are defined by the formula

$$
\left(1-2 r t+r^{2}\right)^{-\lambda}=\sum_{k=0}^{\infty} P_{k}^{\lambda}(t) r^{k}
$$

where $|r|<1,|t| \leq 1$ and $\lambda>0$.

We have [SW, p. 149], if $N>2$,

$$
Z_{\eta}^{k}(\xi)=C_{k, N} P_{k}^{(N-2) / 2}(\xi \cdot \eta) .
$$

It is easy to compute the constants $C_{k, N}$. First, if $\omega_{N-1}$ denotes the Lebesgue measure of $\Sigma_{N-1}$, then

$$
\left\|Z_{\eta}^{k}\right\|_{2}^{2}=\frac{a_{k}}{\omega_{N-1}}
$$

while, on the other hand,

$$
\begin{aligned}
\frac{a_{k}}{\omega_{N-1}} & =C_{k, N}^{2} \int_{\Sigma_{N-1}}\left|P_{k}^{(N-2) / 2}(\xi \cdot \eta)\right|^{2} d \xi \\
& =C_{k, N}^{2} \omega_{N-2} \int_{-1}^{1}\left|P_{k}^{(N-2) / 2}(t)\right|^{2}\left(1-t^{2}\right)^{(N-3) / 2} d t .
\end{aligned}
$$

Now, the polynomials $P_{k}^{(N-2) / 2}(t)$ form an orthogonal basis of

$$
L^{2}\left([-1,1],\left(1-t^{2}\right)^{(N-3) / 2} d t\right)
$$

[SW, p. 151], [AS, p. 774], and

$$
\left\|P_{k}^{(N-2) / 2}\right\|_{2}^{2}=\frac{\pi 2^{4-N} \Gamma(k+N-2)}{k !(2 k+N-2) \Gamma\left(\frac{N-2}{2}\right)^{2}},
$$


where $\Gamma(\cdot)$ denotes the Euler's Gamma function, and, therefore

$$
C_{k, N}^{2}=\frac{a_{k}}{\omega_{N-1} \omega_{N-2}}\left\|P_{k}^{(N-2) / 2}\right\|_{2}^{-2}=\frac{(N+2 k-2)^{2}}{16 \pi^{N}} \Gamma\left(\frac{N-2}{2}\right)^{2} .
$$

Hence

$$
C_{k, N}=\frac{N+2 k-2}{4 \pi^{N / 2}} \Gamma\left(\frac{N-2}{2}\right)
$$

and

$$
Z_{\eta}^{k}(\xi)=\frac{N+2 k-2}{4 \pi^{N / 2}} \Gamma\left(\frac{N-2}{2}\right) P_{k}^{(N-2) / 2}(\xi \cdot \eta) .
$$

The case $N=2$ is slightly different. In this case we can take $P_{k}^{0} \equiv T_{k}$, the Chebyshev's polynomials defined in $[-1,1]$ by

$$
T_{k}(\cos \theta)=\cos k \theta \text {. }
$$

It is known that these polynomials form an orthogonal basis of

$$
L^{2}\left([-1,1],\left(1-t^{2}\right)^{-1 / 2} d t\right)
$$

In this particular case, if $\xi=e^{i \theta}, \eta=e^{i \psi}$, then $\xi \cdot \eta=\cos (\theta-\psi)$, and

$$
\begin{aligned}
& Z_{\eta}^{k}(\xi)=\frac{1}{\pi} \cos k(\theta-\psi)=\frac{1}{\pi} T_{k}(\cos (\theta-\psi)) \\
& =\frac{1}{\pi} P_{k}^{0}(\xi \cdot \eta), \quad k=1,2, \ldots, \\
& Z_{\eta}^{0}(\xi)=\frac{1}{2 \pi}=\frac{1}{2 \pi} P_{0}^{0}(\xi \cdot \eta) \text {. }
\end{aligned}
$$

Therefore,

$$
C_{k, 2}= \begin{cases}\frac{1}{\pi}, & \text { if } k>0, \\ \frac{1}{2 \pi}, & \text { if } k=0 .\end{cases}
$$

We can now write down the expansion of the kernel $\Phi_{\alpha}(|x-y|)$ in a Fourier series of Gegenbauer's polynomials. Fix, first, $\alpha$, with $0<\alpha<N-1$. If we denote by $g(t)$ the function

$$
g(t)=\left(\frac{1}{2-2 t}\right)^{\alpha / 2}
$$

then we can express the kernel $\Phi_{\alpha}$ in terms of $g$ as

$$
\Phi_{\alpha}(|\xi-\eta|)=\Phi_{\alpha}\left(\sqrt{|\xi|^{2}-2 \xi \cdot \eta+|\eta|^{2}}\right)=g(\xi \cdot \eta)
$$


Now, develop $g(t)$ as a Fourier series

$$
g(t)=\sum_{k=0}^{\infty} g_{k} P_{k}^{(N-2) / 2}(t), \quad \text { where } \quad g_{k}\left\|P_{k}^{(N-2) / 2}\right\|_{2}^{2}=\left\langle g, P_{k}^{(N-2) / 2}\right\rangle
$$

and conclude

$$
\Phi_{\alpha}(|\xi-\eta|)=g(\xi \cdot \eta)=\sum_{k=0}^{\infty} g^{k} Z_{\eta}^{k}(\xi)
$$

where $g^{k} C_{k, N}=g_{k}$. Hereafter $F$ will denote the usual hypergeometric function

$$
F(a, b ; c ; t)=\sum_{m=0}^{\infty} \frac{(a)_{m}(b)_{m}}{(c)_{m}} \frac{t^{m}}{m !}
$$

where

$$
(u)_{m}=u(u+1) \ldots(u+m-1)=\frac{\Gamma(u+m)}{\Gamma(u)} .
$$

The polynomials $P_{k}^{(N-2) / 2}$ can be expressed in terms of $F$ [AS, p. 779].

If $N>2$,

$$
P_{k}^{(N-2) / 2}(t)=\left(\begin{array}{c}
k+N-3 \\
k
\end{array}\right) F(-k, k+N-2 ;(N-1) / 2 ;(1-t) / 2) .
$$

Then,

$$
\begin{gathered}
\left\langle g, P_{k}^{(N-2) / 2}\right\rangle=\left(\begin{array}{c}
k+N-3 \\
k
\end{array}\right) \int_{-1}^{1} F(-k, k+N-2 ;(N-1) / 2 ;(1-t) / 2) \\
\cdot(2-2 t)^{-\alpha / 2}\left(1-t^{2}\right)^{(N-3) / 2} d t .
\end{gathered}
$$

Therefore

$$
\begin{aligned}
\left\langle g, P_{k}^{(N-2) / 2}\right\rangle=2^{N-2-\alpha}\left(\begin{array}{c}
k+N-3 \\
k
\end{array}\right) & \int_{0}^{1} s^{-1+(N-1-\alpha) / 2}(1-s)^{-1+(N-1) / 2} \\
& \cdot F(-k, k+N-2 ;(N-1) / 2 ; s) d s .
\end{aligned}
$$

Using the relationship

$$
P_{k}^{(N-2) / 2}(-t)=(-1)^{k} P_{k}^{(N-2) / 2}(t), \quad[\mathbf{S W}, \text { p. 149], [AS, p. 775] }
$$


we have

$$
\begin{aligned}
&\left\langle g, P_{k}^{(N-2) / 2}\right\rangle \\
&=2^{N-2-\alpha}\left(\begin{array}{c}
k+N-3 \\
k
\end{array}\right)(-1)^{k} \int_{0}^{1} s^{-1+(N-1-\alpha) / 2}(1-s)^{-1+(N-1) / 2} \\
& \\
& \cdot F(-k, k+N-2 ;(N-1) / 2 ; 1-s) d s .
\end{aligned}
$$

Term by term integration of the series defining $F$ gives

$$
\int_{0}^{1} s^{a-1}(1-s)^{b-1} F(-k, c ; b ; 1-s) d s=B(a, b) F(-k, c ; a+b ; 1),
$$

where $B(\cdot, \cdot)$ is the Euler's Beta function. Moreover, it is easy to see that ([AS, p. 556])

$$
\begin{aligned}
F(-k, c ; a+b ; 1) & =\frac{\Gamma(a+b) \Gamma(a+b-c+k)}{\Gamma(a+b+k) \Gamma(a+b-c)} \\
& =\frac{\Gamma(a+b)}{\Gamma(a+b+k)}(-1)^{k} \frac{\Gamma(1+c-a-b)}{\Gamma(1+c-a-b-k)}
\end{aligned}
$$

and so

$$
\begin{aligned}
(-1)^{k} \int_{0}^{1} s^{a-1}(1-s)^{b-1} F(-k, c ; b ; 1 & -s) d s \\
& =\frac{\Gamma(a) \Gamma(b) \Gamma(1+c-a-b)}{\Gamma(a+b+k) \Gamma(1+c-a-b-k)} .
\end{aligned}
$$

This gives

$$
\begin{aligned}
& \left\langle g, P_{k}^{(N-2) / 2}\right\rangle \\
& =2^{N-2-\alpha}\left(\begin{array}{c}
k+N-3 \\
k
\end{array}\right) \frac{\Gamma\left(\frac{N-1-\alpha}{2}\right) \Gamma\left(\frac{N-1}{2}\right) \Gamma\left(k+\frac{\alpha}{2}\right)}{\Gamma\left(N-1-\frac{\alpha}{2}+k\right) \Gamma\left(\frac{\alpha}{2}\right)},
\end{aligned}
$$

and

$$
\begin{aligned}
g_{k} & =\frac{\left\langle g, P_{k}^{(N-2) / 2}\right\rangle}{\left\|P_{k}^{(N-2) / 2}\right\|_{2}^{2}} \\
& =2^{N-3-\alpha} \frac{N+2 k-2}{\sqrt{\pi}} \frac{\Gamma\left(\frac{N-1-\alpha}{2}\right) \Gamma\left(\frac{N}{2}-1\right) \Gamma\left(k+\frac{\alpha}{2}\right)}{\Gamma\left(N-1-\frac{\alpha}{2}+k\right) \Gamma\left(\frac{\alpha}{2}\right)} .
\end{aligned}
$$


Therefore,

$$
g^{k}=g_{k} C_{k, N}^{-1}=2^{N-1-\alpha} \pi^{(N-1) / 2} \frac{\Gamma\left(\frac{N-1-\alpha}{2}\right) \Gamma\left(k+\frac{\alpha}{2}\right)}{\Gamma\left(N-1-\frac{\alpha}{2}+k\right) \Gamma\left(\frac{\alpha}{2}\right)},
$$

if $N>2$. On the other hand, if $N=2$, the $k$-th Chebyshev's polynomial is $T_{k}(t)=F(-k, k ; 1 / 2 ;(1-t) / 2),($ see $[\mathbf{A S}$, p. 779$])$, and

$$
\left\langle g, P_{k}^{0}\right\rangle=\int_{-1}^{1}(2-2 t)^{-\alpha / 2} F(-k, k ; 1 / 2 ;(1-t) / 2)\left(1-t^{2}\right)^{-1 / 2} d t .
$$

Using the above computations when $N=2$, we have that

$$
\left\langle g, P_{k}^{0}\right\rangle=2^{-\alpha} \pi^{1 / 2} \frac{\Gamma\left(\frac{1-\alpha}{2}\right) \Gamma\left(k+\frac{\alpha}{2}\right)}{\Gamma\left(1-\frac{\alpha}{2}+k\right) \Gamma\left(\frac{\alpha}{2}\right)} .
$$

Moreover it is easy to see, [AS, p. 774], that

$$
\left\|P_{k}^{0}\right\|_{2}^{2}= \begin{cases}\frac{\pi}{2}, & \text { if } k>0 \\ \pi, & \text { if } k=0\end{cases}
$$

and also that $C_{k, 2}^{-1}=2\left\|P_{k}^{0}\right\|_{2}^{2}$.

Then

$$
g^{k}=\frac{\left\langle g, P_{k}^{0}\right\rangle}{\left\|P_{k}^{0}\right\|_{2}^{2}} C_{k, 2}^{-1},
$$

and so $(2)$ is also satisfied in this case $(N=2)$. Therefore we have proved the following:

Lemma 1. For all $N \in \mathbb{N}, N>1$ and $0<\alpha<N-1$,

$$
\Phi_{\alpha}(|\xi-\eta|)=\sum_{k=0}^{\infty} g^{k} Z_{\eta}^{k}(\xi)
$$

where

$$
g^{k}=2^{N-1-\alpha} \pi^{(N-1) / 2} \frac{\Gamma\left(\frac{N-1-\alpha}{2}\right) \Gamma\left(k+\frac{\alpha}{2}\right)}{\Gamma\left(N-1-\frac{\alpha}{2}+k\right) \Gamma\left(\frac{\alpha}{2}\right)} .
$$

Now we can express the $\alpha$-energy of a measure $\mu$ in terms of its Poisson extension $P_{\mu}$. 
Lemma 2. If $\mu$ is a signed measure supported on $\Sigma_{N-1}$, we have:

(i) If $0<\alpha<N-1$, then

$$
I_{\alpha}(\mu)=C(N, \alpha) \int_{0}^{1}\left\{\int_{\Sigma_{N-1}}\left|P_{\mu}(r \xi)\right|^{2} d \xi\right\} r^{\alpha-1}\left(1-r^{2}\right)^{N-2-\alpha} d r
$$

with

$$
C(N, \alpha)=\frac{4 \pi^{N / 2}}{\Gamma\left(\frac{\alpha}{2}\right) \Gamma\left(\frac{N-\alpha}{2}\right)} .
$$

(ii) If $m=\mu\left(\Sigma_{N-1}\right)$, then

$$
\begin{aligned}
I_{0}(\mu)= & \omega_{N-1} \int_{0}^{1} \int_{\Sigma_{N-1}}\left|P_{\mu}(r \xi)-\frac{m}{\omega_{N-1}}\right|^{2} d \xi\left(1-r^{2}\right)^{N-2} \frac{d r}{r} \\
& +\frac{m^{2}}{2}\left[\frac{\Gamma^{\prime}}{\Gamma}\left(\frac{N}{2}\right)-\frac{\Gamma^{\prime}}{\Gamma}(N-1)\right] .
\end{aligned}
$$

In particular, if $N=2$,

$$
I_{0}(\mu)=2 \pi \int_{0}^{1} \int_{0}^{2 \pi}\left|P_{\mu}\left(r e^{i \theta}\right)-\frac{m}{2 \pi}\right|^{2} d \theta \frac{d r}{r} .
$$

Proof. Let $\left\{\mu_{j}^{k}\right\}, k \geq 0,1 \leq j \leq a_{k}$, be the Fourier coefficientes of $\mu$, i.e.,

$$
\mu \sim \sum_{k=0}^{\infty} \sum_{j=1}^{a_{k}} \mu_{j}^{k} Y_{j}^{k}
$$

Recall that $P_{\mu}$ is defined by

$$
P_{\mu}(r \xi)=\int_{\Sigma_{N-1}} p(\eta, r \xi) d \mu(\eta)
$$

where $p(\eta, r \xi)$ is the classical (normalized) Poisson kernel

$$
p(\eta, r \xi)=\frac{1}{\omega_{N-1}} \frac{1-r^{2}}{|\eta-r \xi|^{N}} .
$$

We have [SW, p. 145]

$$
p(\eta, r \xi)=\sum_{k=0}^{\infty} r^{k} Z_{\eta}^{k}(\xi)=\sum_{k, j} r^{k} Y_{j}^{k}(\eta) Y_{j}^{k}(\xi) .
$$


Now, Plancherel's theorem gives that

$$
P_{\mu}(r \xi)=\sum_{k, j} r^{k} \mu_{j}^{k} Y_{j}^{k}(\xi)
$$

Using again Plancherel's theorem we obtain that

$$
\int_{\Sigma_{N-1}}\left|P_{\mu}(r \xi)\right|^{2} d \xi=\sum_{k, j} r^{2 k}\left|\mu_{j}^{k}\right|^{2}
$$

and so if we denote by $\Lambda$ the right hand side in (i), we have that

$$
\Lambda=C(N, \alpha) \sum_{k, j}\left|\mu_{j}^{k}\right|^{2} \int_{0}^{1} r^{2 k+\alpha-1}\left(1-r^{2}\right)^{N-2-\alpha} d r
$$

and, substituting $r^{2}=t$, we get that

$$
\Lambda=\frac{C(N, \alpha)}{2} \sum_{k, j} \frac{\Gamma\left(k+\frac{\alpha}{2}\right) \Gamma(N-1-\alpha)}{\Gamma\left(k+N-1-\frac{\alpha}{2}\right)}\left|\mu_{j}^{k}\right|^{2}=\sum_{\jmath, k} g^{k}\left|\mu_{\jmath}^{k}\right|^{2}
$$

Note that we have used the known duplication formula for the Gamma function in the last equality.

On the other hand, by (1),

$$
\Phi_{\alpha}(|\xi-\eta|)=\sum_{k=0}^{\infty} g^{k} Z_{\eta}^{k}(\xi)=\sum_{k, j} g^{k} Y_{j}^{k}(\eta) Y_{\jmath}^{k}(\xi),
$$

and using Plancherel's theorem we obtain that

$$
\begin{gathered}
\int_{\Sigma_{N-1}} \Phi_{\alpha}(|\xi-\eta|) d \mu(\eta)=\sum_{k, j} g^{k} \mu_{j}^{k} Y_{j}^{k}(\xi) \\
I_{\alpha}(\mu)=\sum_{k, j} g^{k}\left|\mu_{j}^{k}\right|^{2}=\Lambda
\end{gathered}
$$

This finishes the proof of (i).

In order to prove (ii) observe that

$$
\int_{\Sigma_{N-1}}\left|P_{\mu}(r \xi)-\frac{m}{\omega_{N-1}}\right|^{2} d \xi+\frac{m^{2}}{\omega_{N-1}}=\int_{\Sigma_{N-1}}\left|P_{\mu}(r \xi)\right|^{2} d \xi .
$$

Integrating this equality we have that

$$
\begin{aligned}
I_{\alpha}(\mu)= & C(N, \alpha) \int_{0}^{1} \int_{\Sigma_{N-1}}\left|P_{\mu}(r \xi)-\frac{m}{\omega_{N-1}}\right|^{2} d \xi r^{\alpha-1}\left(1-r^{2}\right)^{N-2-\alpha} d r \\
& +m^{2} U(\alpha)
\end{aligned}
$$


where

$$
U(\alpha)=\frac{\Gamma(N / 2) \Gamma(N-1-\alpha)}{\Gamma((N-\alpha) / 2) \Gamma(N-1-\alpha / 2)}
$$

and hence

$$
\begin{aligned}
\lim _{\alpha \rightarrow 0} \frac{I_{\alpha}(\mu)-m^{2} U(\alpha)}{\alpha} & \\
& =\omega_{N-1} \int_{0}^{1} \int_{\Sigma_{N-1}}\left|P_{\mu}(r \xi)-\frac{m}{\omega_{N-1}}\right|^{2} d \xi\left(1-r^{2}\right)^{N-2} \frac{d r}{r} .
\end{aligned}
$$

On the other hand,

$$
\begin{aligned}
\lim _{\alpha \rightarrow 0} \frac{I_{\alpha}(\mu)-m^{2} U(\alpha)}{\alpha} & =\lim _{\alpha \rightarrow 0} \frac{I_{\alpha}(\mu)-m^{2}}{\alpha}-m^{2} \lim _{\alpha \rightarrow 0} \frac{U(\alpha)-1}{\alpha} \\
& =I_{0}(\mu)-m^{2} U^{\prime}(0)
\end{aligned}
$$

and

$$
U^{\prime}(0)=\frac{1}{2}\left[\frac{\Gamma^{\prime}}{\Gamma}\left(\frac{N}{2}\right)-\frac{\Gamma^{\prime}}{\Gamma}(N-1)\right] .
$$

This finishes the proof of Lemma 2.

\section{Distortion of $\alpha$-capacity.}

We need the following lemmas.

Lemma 3. Let $\mu$ be a finite positive measure in $\partial \Delta$, and let $f$ be an inner function. Then, there exists a unique positive measure $\widetilde{\nu}$ in $\mathbb{S}_{n}$ such that $P_{\mu} \circ f=P_{\widetilde{\nu}}$ and

$$
\widetilde{\nu}\left(f^{-1}(\operatorname{support} \mu)\right)=\widetilde{\nu}\left(\mathbb{S}_{n}\right) .
$$

Moreover, if $f(0)=0$, then

$$
\frac{1}{\omega_{2 n-1}} \widetilde{\nu}\left(\mathbb{S}_{n}\right)=\frac{1}{2 \pi} \mu(\partial \Delta)
$$

Proof. It is essentially the same proof as that of Lemma 1 of $[\mathbf{F P}]$, but see Lemma 10 below for further details.

A different normalization is useful; choosing $\nu=\left(2 \pi / \omega_{2 n-1}\right) \widetilde{\nu}$, one obtaj

$$
P_{\nu}=\frac{2 \pi}{\omega_{2 n-1}} P_{\mu} \circ f \quad \text { and } \quad \nu\left(\mathbb{S}_{n}\right)=\mu(\partial \Delta) .
$$

The following is well known 
Lemma 4. (Subordination principle). Let $f: \mathbb{B}_{n} \longrightarrow \Delta$ be a holomorphic function such that $f(0)=0$, and let $v: \Delta \longrightarrow \mathbb{R}$ be a subharmonic function. Then

$$
\frac{1}{\omega_{2 n-1}} \int_{\mathbb{S}_{n}} v(f(r \xi)) d \xi \leq \frac{1}{2 \pi} \int_{0}^{2 \pi} v\left(r e^{\imath \theta}\right) d \theta .
$$

It will be relevant later on to recall the well known fact that, in the case $n=1$, equality in Lemma 4 holds for a given $r, 0<r<1$, if and only if either $v$ is harmonic in $\Delta_{r}=\{|z|<r\}$ or $f$ is a rotation. Note also that there is no such equality statement when $n>1$ since in higher dimensions the extremal functions in Schwarz's lemma are not so clearly determined (see e.g. [R, p. 164]).

Lemma 5. Let $\mu$ be a signed measure on $\partial \Delta, f$ an inner function with $f(0)=0$, and $\nu$ a signed measure on $\mathbb{S}_{n}$ such that

$$
P_{\nu}=\left(2 \pi / \omega_{2 n-1}\right) P_{\mu} \circ f
$$

Then

(i) If $n=1$ and $0 \leq \alpha<1$, then

$$
I_{\alpha}(\nu) \leq I_{\alpha}(\mu) .
$$

(ii) If $n>1$ and $0<\alpha<1$, then

$$
I_{2 n-2+\alpha}(\nu) \leq K(n, \alpha) I_{\alpha}(\mu)
$$

where

$$
K(n, \alpha)=\frac{(n-1) ! \Gamma\left(\frac{\alpha}{2}\right)}{\Gamma\left(n-1+\frac{\alpha}{2}\right)} .
$$

If $\alpha=0$ and $m=\mu(\partial \Delta)=\nu\left(\mathbb{S}_{n}\right)$, we have

$$
I_{2 n-2}(\nu) \leq(2 n-2) I_{0}(\mu)+m^{2} .
$$

The measure $\nu$ is obtained from Lemma 3 by splitting $\mu$ into its positive and negative parts. Note that for fixed $\alpha$,

$$
K(n, \alpha) \sim n^{1-\alpha / 2} \Gamma\left(\frac{\alpha}{2}\right), \quad \text { as } n \rightarrow \infty,
$$

while for fixed $n>1$

$$
K(n, \alpha) \sim \frac{C_{n}}{\alpha}, \quad \text { as } \alpha \rightarrow 0
$$


Let us observe also that $K(n, \alpha)$ takes the value 1 for $n=1$.

Proof. Since $\left|P_{\mu}-\frac{m}{2 \pi}\right|^{2}$ and $\left|P_{\mu}\right|^{2}$ are subharmonic, we obtain by subordination, Lemma 4 , that if $n=1$ and $\alpha=0$

$$
\int_{0}^{2 \pi}\left|P_{\nu}-\frac{m}{2 \pi}\right|^{2} d \theta=\int_{0}^{2 \pi}\left|P_{\mu}(f)-\frac{m}{2 \pi}\right|^{2} d \theta \leq \int_{0}^{2 \pi}\left|P_{\mu}-\frac{m}{2 \pi}\right|^{2} d \theta,
$$

and if $n \geq 1,0<\alpha<1$, that

$$
\int_{\mathbb{S}_{n}}\left|P_{\nu}\right|^{2} d \xi=\left(\frac{2 \pi}{\omega_{2 n-1}}\right)^{2} \int_{\mathbb{S}_{n}}\left|P_{\mu}(f)\right|^{2} d \xi \leq \frac{2 \pi}{\omega_{2 n-1}} \int_{0}^{2 \pi}\left|P_{\mu}\right|^{2} d \theta
$$

In the first case, we obtain

$$
I_{0}(\nu) \leq I_{0}(\mu)
$$

by integrating with respect to $2 \pi d r / r$ and applying Lemma 2, part (ii).

In the second case, using Lemma 2, part (i), and Lemma 4 with $v=\left|P_{\mu}\right|^{2}$, we have that

$$
\begin{aligned}
I_{2 n-2+\alpha}(\nu)= & C(2 n, 2 n-2+\alpha) \int_{0}^{1}\left\{\int_{\mathbb{S}_{n}}\left|P_{\nu}(r \xi)\right|^{2} d \xi\right\} r^{2 n-2+\alpha-1} \frac{d r}{\left(1-r^{2}\right)^{\alpha}} \\
\leq & \frac{C(2 n, 2 n-2+\alpha)}{C(2, \alpha)} C(2, \alpha) \\
& \cdot \frac{2 \pi}{\omega_{2 n-1}} \int_{0}^{1}\left\{\int_{0}^{2 \pi}\left|P_{\mu}\left(r e^{i \theta}\right)\right|^{2} d \theta\right\} r^{\alpha-1} \frac{d r}{\left(1-r^{2}\right)^{\alpha}} \\
= & K(n, \alpha) I_{\alpha}(\mu),
\end{aligned}
$$

where

$$
K(n, \alpha)=\frac{(n-1) ! \Gamma\left(\frac{\alpha}{2}\right)}{\Gamma\left(n-1+\frac{\alpha}{2}\right)} .
$$

Finally, since $\nu\left(\mathbb{S}_{n}\right)=m$,

$$
\int_{\mathbb{S}_{n}}\left|P_{\nu}(r \xi)-\frac{m}{\omega_{2 n-1}}\right|^{2} d \xi=\int_{\mathbb{S}_{n}}\left|P_{\nu}(r \xi)\right|^{2} d \xi-\frac{m^{2}}{\omega_{2 n-1}}
$$

and so, Lemma 2 gives, if $n>1$, that

$$
I_{2 n-2}(\nu)=m^{2}+\frac{4 \pi^{n}}{(n-2) !} \int_{0}^{1} \int_{\mathbb{S}_{n}}\left|P_{\nu}(r \xi)-\frac{m}{\omega_{2 n-1}}\right|^{2} d \xi r^{2 n-3} d r .
$$


By Lemmas 3 and 4, we get that

$$
\begin{aligned}
\int_{\mathbb{S}_{n}}\left|P_{\nu}(r \xi)-\frac{m}{\omega_{2 n-1}}\right|^{2} d \xi & =\int_{\mathbb{S}_{n}}\left|\frac{2 \pi}{\omega_{2 n-1}} P_{\mu}(f(r \xi))-\frac{m}{\omega_{2 n-1}}\right|^{2} d \xi \\
& =\left(\frac{2 \pi}{\omega_{2 n-1}}\right)^{2} \int_{\mathbb{S}_{n}}\left|P_{\mu}(f(r \xi))-\frac{m}{2 \pi}\right|^{2} d \xi \\
& \leq \frac{2 \pi}{\omega_{2 n-1}} \int_{0}^{2 \pi}\left|P_{\mu}\left(r e^{i \theta}\right)-\frac{m}{2 \pi}\right|^{2} d \theta
\end{aligned}
$$

Therefore

$$
\begin{aligned}
I_{2 n-2}(\nu) & \leq m^{2}+\frac{4 \pi^{n}}{(n-2) !} \int_{0}^{1} \frac{2 \pi}{\omega_{2 n-1}} \int_{0}^{2 \pi}\left|P_{\mu}\left(r e^{i \theta}\right)-\frac{m}{2 \pi}\right|^{2} d \theta \frac{d r}{r} \\
& =m^{2}+\frac{4 \pi^{n}}{(n-2) !} \frac{1}{\omega_{2 n-1}} I_{0}(\mu) \\
& =m^{2}+(2 n-2) I_{0}(\mu) .
\end{aligned}
$$

The proof of Lemma 5 is finished.

Finally, we can prove

Theorem 1. If $f$ is inner in the unit disk $\Delta, f(0)=0$, and $E$ is a Borel subset of $\partial \Delta$, we have:

$$
\operatorname{cap}_{\alpha}\left(f^{-1}(E)\right) \geq \operatorname{cap}_{\alpha}(E), \quad 0 \leq \alpha<1 .
$$

Moreover, if $E$ is any Borel subset of $\partial \Delta$ with $\operatorname{cap}_{\alpha}(E)>0$, equality holds if and only if either $f$ is a rotation or $\operatorname{cap}_{\alpha}(E)=\operatorname{cap}_{\alpha}(\partial \Delta)$.

Notice the following consequence concerning invariant sets. It is well known that an inner function $f$ with $f(0)=0$, which is not a rotation, is ergodic with respect to Lebesgue measure, see e.g. $[\mathbf{P}]$. As a consequence of the above, it is also ergodic with respect to $\alpha$-capacity. More precisely,

Corollary. With the hypotheses of Theorem 1 , if $f$ is not a rotation and if the symmetric difference between $E$ and $f^{-1}(E)$ has zero $\alpha$-capacity, then either $\operatorname{cap}_{\alpha}(E)=0$ or $\operatorname{cap}_{\alpha}(E)=\operatorname{cap}_{\alpha}(\partial \Delta)$.

In higher dimensions we have

Theorem 2. If $f$ is inner in the unit ball of $\mathbb{C}^{n}, f(0)=0$, and $E$ is a Borel subset of $\partial \Delta$, we have:

$$
\operatorname{cap}_{2 n-2+\alpha}\left(f^{-1}(E)\right) \geq K(n, \alpha)^{-1} \operatorname{cap}_{\alpha}(E), \quad 0<\alpha<1
$$


and

$$
\frac{1}{\operatorname{cap}_{2 n-2}\left(f^{-1}(E)\right)} \leq 1+(2 n-2) \log \frac{1}{\operatorname{cap}_{0}(E)}, \quad(n>1)
$$

Proof of Theorems 1 and 2 . To prove the inequalities in the theorems we may assume that $E$ is closed. Assume first that $n=1,0<\alpha<1$. Let us denote by $\mu_{e}$ the $\alpha$-equilibrium probability distribution of $E$, and let $\nu$ be the probability measure such that $P_{\nu}=P_{\mu_{e}} \circ f$. By Lemma 5,

$$
I_{\alpha}(\nu) \leq I_{\alpha}\left(\mu_{e}\right)=\left(\operatorname{cap}_{\alpha}(E)\right)^{-1} .
$$

But, from Lemma $3, \nu\left(f^{-1}(E)\right)=1$, and so

$$
I_{\alpha}(\nu)=\iint_{f^{-1}(E) \times f^{-1}(E)} \Phi_{\alpha}(|z-w|) d \nu(z) d \nu(w) .
$$

Now, let $\left\{K_{n}\right\}$ be an increasing sequence of compacts subsets in $\partial \Delta, K_{n} \subset$ $f^{-1}(E)$, such that $\nu\left(K_{n}\right) \nearrow 1$. Then, for each $n \geq 1$,

$$
\begin{aligned}
I_{\alpha}(\nu) & =\iint_{f^{-1}(E) \times f^{-1}(E)} \Phi_{\alpha}(|z-w|) d \nu(z) d \nu(w) \\
& \geq \nu\left(K_{n}\right)^{2} \iint_{K_{n} \times K_{n}} \Phi_{\alpha}(|z-w|) \frac{d \nu(z)}{\nu\left(K_{n}\right)} \frac{d \nu(w)}{\nu\left(K_{n}\right)} \\
& \geq \nu\left(K_{n}\right)^{2}\left(\operatorname{cap}_{\alpha}\left(K_{n}\right)\right)^{-1} \\
& \geq \nu\left(K_{n}\right)^{2}\left(\operatorname{cap}_{\alpha}\left(f^{-1}(E)\right)\right)^{-1}
\end{aligned}
$$

and consequently

$$
I_{\alpha}(\nu) \geq\left(\operatorname{cap}_{\alpha}\left(f^{-1}(E)\right)\right)^{-1} .
$$

The inequality in Theorem 1 follows now from (4) and (5).

The cases $\underline{n>1}$ (Theorem 2) and $\underline{n=1, \alpha=0}$ are completely analogous.

Proof of the equality statement of Theorem 1. First we prove it assuming that $E$ is closed, to show the ideas that we will use to demonstrate the general case.

Suppose that $0<\alpha<1$. We have seen that

$$
\frac{1}{\operatorname{cap}_{\alpha}\left(f^{-1}(E)\right)} \leq I_{\alpha}(\nu) \leq I_{\alpha}\left(\mu_{e}\right)=\frac{1}{\operatorname{cap}_{\alpha}(E)} .
$$

Therefore, if $E$ and $f^{-1}(E)$ have the same $\alpha$-capacity, then

$$
I_{\alpha}(\nu)=I_{\alpha}\left(\mu_{e}\right),
$$


and this is possible only if for all $r \in(0,1)$,

$$
\int_{0}^{2 \pi}\left|P_{\mu_{e}}\left(r e^{i \theta}\right)\right|^{2} d \theta=\int_{0}^{2 \pi}\left|P_{\mu_{e}}\left(f\left(r e^{i \theta}\right)\right)\right|^{2} d \theta .
$$

This can occur only if either $f$ is a rotation or $\left|P_{\mu_{e}}\right|^{2}$ is harmonic. In the latter case, we obtain that $\mu_{e}$ is normalized Lebesgue measure, or equivalently that $\operatorname{cap}_{\alpha}(E)=\operatorname{cap}_{\alpha}(\partial \Delta)$. Since $E$ is closed, it follows that $E=\partial \Delta$.

In order to prove the general case we need a characterization of the $\alpha$ capacity of $E$ when $E$ is not closed (see Lemma 6 below). We begin by recalling some facts about convergence of measures.

We will say that a sequence of signed measures $\left\{\sigma_{n}\right\}$ with supports contained in a compact set $K$ converges $w^{*}$ to a signed measure $\sigma$ if

$$
\int h(x) d \sigma_{n}(x) \underset{n \rightarrow \infty}{\rightarrow} \int h(x) d \sigma(x), \quad \text { for all } h \in C(K) .
$$

Here, the $w^{*}$-convergence refers to the duality between the space of signed measures on $K$ and the space $C(K)$ of continuous functions with support contained in $K$.

In this Section, we will denote by $\mathcal{M}_{\alpha}(K)(0 \leq \alpha<1)$ the vector space of all signed measures whose support is contained in the set $K$ and whose $\alpha$-energy is finite. $\mathcal{M}_{\alpha}(\mathbb{C})$ or $\mathcal{M}_{\alpha}(\bar{\Delta})$ is denoted simply by $\mathcal{M}_{\alpha}$, and $\mathcal{M}_{\alpha}^{+}$ denotes the corresponding cone of positive measures.

The positivity properties of $I_{\alpha}[\mathbf{L}$, p. 79-80] allow us to define an inner product in $\mathcal{M}_{\alpha}$ (for $\left.0<\alpha<1\right)$ and e.g. in $\mathcal{M}_{0}(\{|z|=1 / 2\}$ ) (for $\alpha=0$ ) as follows

$$
\langle\sigma, \gamma\rangle=\iint \Phi_{\alpha}(|x-y|) d \sigma(x) d \gamma(y)
$$

Observe that the associated norm verifies

$$
\|\sigma\|^{2}=I_{\alpha}(\sigma) .
$$

In the next lemma we collect some useful information concerning the above inner product.

\section{Lemma 6.}

(i) If $0<\alpha<1, K$ is a compact subset of $\mathbb{C},\left\{\sigma_{n}\right\}$ is a Cauchy sequence (with respect to the inner product) in $\mathcal{M}_{\alpha}^{+}(K)$ and $\sigma_{n} \stackrel{w^{*}}{\rightarrow} \sigma$, then

$$
\left\|\sigma_{n}-\sigma\right\| \longrightarrow 0, \quad \text { as } n \rightarrow \infty \text {. }
$$

(ii) If $E$ is any Borel subset of $K$, then

$$
\frac{1}{\operatorname{cap}_{\alpha}(E)}=\inf \left\{I_{\alpha}(\mu): \quad \mu \text { a probability measure, } \mu(E)=1\right\}
$$


and there exists a probability measure $\mu_{e}$ supported on $\bar{E}$ such that

$$
\frac{1}{\operatorname{cap}_{\alpha}(E)}=I_{\alpha}\left(\mu_{e}\right)
$$

In fact, if $K_{n}$ is an increasing sequence of compact subsets of E such that

$$
\operatorname{cap}_{\alpha}\left(K_{n}\right) \nearrow \operatorname{cap}_{\alpha}(E)
$$

and if $\mu_{n}$ is the equilibrium distribution of $K_{n}$, then

$$
\mu_{n} \stackrel{w^{*}}{\rightarrow} \mu_{e} \quad \text { and } \quad\left\|\mu_{n}-\mu_{e}\right\| \longrightarrow 0
$$

as $n \rightarrow \infty$.

These statements remain true in the case $\alpha=0$, if $K$ is a compact subset of $\Delta$.

Lemma 6 is contained in $[\mathbf{L}$, p. $82,89,145]$ if $0<\alpha<1$. The case $\alpha=0$ is similar, though we need the restriction $K \subset \Delta$ so that $\|\cdot\|$ is a norm [L, p. 80].

Now we are ready to finish the proof of Theorem 1. Let $E$ be a Borel subset of $\partial \Delta$ such that

$$
\operatorname{cap}_{\alpha}\left(f^{-1}(E)\right)=\operatorname{cap}_{\alpha}(E)>0 .
$$

We choose an increasing sequence of compact sets $K_{n} \subset E$ such that $\operatorname{cap}_{\alpha}\left(K_{n}\right) \nearrow \operatorname{cap}_{\alpha}(E)$. Let $\mu_{n}$ be the $\alpha$-equilibrium measure of $K_{n}$ and let $\mu_{e}$ be the probability measure supported on $\bar{E}$ given by Lemma 6 . We have

$$
\mu_{n} \stackrel{w^{*}}{\rightarrow} \mu_{e} \quad \text { and } \quad I_{\alpha}\left(\mu_{n}\right) \searrow I_{\alpha}\left(\mu_{e}\right),
$$

as $n \rightarrow \infty$. In fact,

$$
\left\|\mu_{n}-\mu_{e}\right\| \rightarrow 0, \quad \text { as } n \rightarrow \infty .
$$

Let $\nu_{n}$ be the probability measure, with $\nu_{n}\left(f^{-1}\left(K_{n}\right)\right)=1$, such that $P_{\nu_{n}}=$ $P_{\mu_{n}} \circ f$ (see Lemma 3). We can suppose after extracting a subsequence if necessary, that $\nu_{n}$ converges $w^{*}$ to a probability measure $\nu$ on $\overline{f^{-1}(E)}$. Since the Poisson kernel is continuous in $\Delta$ we obtain, by using the $w^{*}$-convergence, that

$$
P_{\mu_{n}} \rightarrow P_{\mu_{e}} \text { and } P_{\nu_{n}} \rightarrow P_{\nu}, \quad \text { as } n \rightarrow \infty,
$$

pointwise. Therefore $P_{\nu}=P_{\mu_{e}} \circ f$, which in particular shows that $\nu$ is a probability measure supported on $f^{-1}(\bar{E})$. 
Claim. $I_{\alpha}\left(\nu_{n}\right) \rightarrow I_{\alpha}(\nu)$ as $n \rightarrow \infty$.

Since $\nu_{n}$ is a probability measure on $f^{-1}(E)$, Lemma 6 guarantees that

$$
\frac{1}{\operatorname{cap}_{\alpha}\left(f^{-1}(E)\right)} \leq I_{\alpha}\left(\nu_{n}\right)
$$

and so, by letting $n \rightarrow \infty$, and using that $P_{\nu}=P_{\mu_{e}} \circ f$ (by Lemma 5) we obtain that

$$
\frac{1}{\operatorname{cap}_{\alpha}\left(f^{-1}(E)\right)} \leq I_{\alpha}(\nu) \leq I_{\alpha}\left(\mu_{e}\right)=\frac{1}{\operatorname{cap}_{\alpha}(E)}
$$

From (6), we deduce that $I_{\alpha}(\nu)=I_{\alpha}\left(\mu_{e}\right)$. Finally, we can reason as in the case of $E$ being closed and conclude that either $f$ is a rotation or $\mu_{e}$ is normalized Lebesgue measure, i.e., $\operatorname{cap}_{\alpha}(E)=\operatorname{cap}_{\alpha}(\partial \Delta)$.

Proof of the Claim. Consider first the case $0<\alpha<1$. Since $P_{\nu_{p}-\nu_{n}}=$ $P_{\mu_{p}-\mu_{n}} \circ f$, by Lemma 5 we obtain that

$$
\left\|\nu_{p}-\nu_{n}\right\|^{2}=I_{\alpha}\left(\nu_{p}-\nu_{n}\right) \leq I_{\alpha}\left(\mu_{p}-\mu_{n}\right)=\left\|\mu_{p}-\mu_{n}\right\|^{2} \underset{p, n \rightarrow \infty}{\rightarrow} 0 .
$$

Therefore $\left\{\nu_{n}\right\}$ is a Cauchy sequence in the norm and so, by Lemma 6 , we have that

$$
\left\|\nu_{n}-\nu\right\| \rightarrow 0 \quad \text { and } \quad I_{\alpha}\left(\nu_{n}\right) \rightarrow I_{\alpha}(\nu)
$$

as $n \rightarrow \infty$.

For $\lambda>0$, and $A \subset \mathbb{C}$, we will denote by $\lambda A$ the set $\lambda A=\{\lambda z: z \in A\}$.

If $E$ is a Borel subset of $\partial \Delta$, then $\frac{1}{2} E$ is a Borel subset of $\{|z|=1 / 2\}$. Also, if $\sigma$ is a probability measure in $\partial \Delta$, we will denote by $\sigma^{*}$ the probability measure in $\{|z|=1 / 2\}$ defined by

$$
\sigma(A)=\sigma^{*}\left(\frac{1}{2} A\right)
$$

for $A$ a Borel subset of $\partial \Delta$. It is clear that

$$
I_{0}\left(\sigma^{*}\right)=I_{0}(\sigma)+\log 2 .
$$

Now, in order to prove the case $\alpha=0$, let $\mu_{n}^{*}$ and $\nu_{n}^{*}$ be the measures defined from $\mu_{n}$ and $\nu_{n}$ by (7). Then using again Lemma 5 and (8) we have that

$$
\begin{aligned}
\left\|\nu_{p}^{*}-\nu_{n}^{*}\right\|^{2} & =I_{0}\left(\nu_{p}^{*}-\nu_{n}^{*}\right)=I_{0}\left(\nu_{p}-\nu_{n}\right)+\log 2 \\
& \leq I_{0}\left(\mu_{p}-\mu_{n}\right)+\log 2=\left\|\mu_{p}^{*}-\mu_{n}^{*}\right\|_{p, n \rightarrow \infty}^{2} 0 .
\end{aligned}
$$


Therefore $\left\{\nu_{n}^{*}\right\}$ is a Cauchy sequence in the norm and again by Lemma 6 , we obtain that

$$
\left\|\nu_{n}^{*}-\nu^{*}\right\| \rightarrow 0 \quad \text { and } \quad I_{0}\left(\nu_{n}^{*}\right) \rightarrow I_{0}\left(\nu^{*}\right)
$$

as $n \rightarrow \infty$. It follows, from (8) that

$$
I_{0}\left(\nu_{n}\right) \rightarrow I_{0}(\nu), \quad \text { as } n \rightarrow \infty .
$$

\section{Some further results on distortion of capacity in the case $\mathbf{n}=\mathbf{1}$.}

First we show that Theorem 1 is sharp. In what follows $|\cdot|$ will denote not normalized Lebesgue measure in $\partial \Delta$ ( i.e. $|\partial \Delta|=2 \pi$ ).

Proposition 1. $\operatorname{cap}_{\alpha}\left(f^{-1}(E)\right)$ can take any value between $\operatorname{cap}_{\alpha}(E)$ and $\operatorname{cap}_{\alpha}(\partial \Delta)$. More precisely, given $0<s \leq t<\operatorname{cap}_{\alpha}(\partial \Delta)$ there exist a Borel subset $E$ of $\partial \Delta$ and an inner function $f$ with $f(0)=0$ such that $\operatorname{cap}_{\alpha}(E)=s$ and $\operatorname{cap}_{\alpha}\left(f^{-1}(E)\right)=t$.

In order to prove this, we need the following lemma whose proof will given later.

Lemma 7. Let $I$ be any closed interval in $\partial \Delta$ with $|I|>0$, and let $B$ be a finite union of closed intervals in $\partial \Delta$ such that $|B|=|I|$. Then there exists an inner function $f$ such that

$$
f(0)=0 \quad \text { and } \quad f^{-1}(I)=B .
$$

In fact, if $0<|I|<2 \pi$, then $f$ is unique.

Remark. It is natural to wonder if this lemma holds in higher dimensions, more precisely: Is it true that given an interval $I$ in $\partial \Delta$ and a Borel subset $B$ of $\mathbb{S}_{n}$ such that

$$
\frac{|B|}{\omega_{2 n-1}}=\frac{|I|}{2 \pi}
$$

there is an inner function $f: \mathbb{B}_{n} \longrightarrow \Delta$ such that $f^{-1}(I) \stackrel{\circ}{=} B$ ?

It is not possible to construct such $f$ by using the Ryll-Wojtaszczyk polynomials (see $[\mathbf{R} 1]$ ), since in that case the following stronger result would be true too: 
Given $E, I$ subsets of $\partial \Delta$ with $|E|=|I|$ and $N \in \mathbb{N}$, there exists an inner function $f: \Delta \longrightarrow \Delta$ such that

$$
E=f^{-1}(I), \quad \text { and } \quad f^{(j)}(0)=0, \quad \text { if } j \leq N .
$$

But it is easy to see, as a consequence of Lemma 8, that in general this is not possible.

Proof of Proposition 1. Let $I$ be a closed interval in $\partial \Delta$ centered at 1 and such that $\operatorname{cap}_{\alpha}(I)=s$. Consider the function $g(z)=z^{2}$. Then (see e.g. [FP] or Proposition 3 below),

$$
s=\operatorname{cap}_{\alpha}(I)<\operatorname{cap}_{\alpha}\left(g^{-1}(I)\right)<\cdots<\operatorname{cap}_{\alpha}\left(g^{-k}(I)\right) \underset{k \rightarrow \infty}{\rightarrow} \operatorname{cap}_{\alpha}(\partial \Delta) .
$$

Therefore, if $t=\operatorname{cap}_{\alpha}\left(g^{-k}(I)\right)$ for some $k$, we are done.

Note that $g^{-k}(I)$ consists of $2^{k}$ closed intervals of length $|I| / 2^{k}$ and centered at the points $z_{j, k}=e^{2 \pi j i / 2^{k}}\left(j=1, \ldots, 2^{k}\right)$.

If $\operatorname{cap}_{\alpha}\left(g^{-(k-1)}(I)\right)<t<\operatorname{cap}_{\alpha}\left(g^{-k}(I)\right)$ a simple continuity argument shows that there exist a finite union $B$ of $2^{k}$ closed intervals in $\partial \Delta$ of total length $|I|$ with $\operatorname{cap}_{\alpha}(B)=t$.

Finally, applying Lemma 7 to the pair $I, B$ we obtain an inner function $f$ with $f(0)=0$ and $f^{-1}(I)=B$.

Proof of Lemma 7. Let $u$ be the Poisson integral of the characteristic function of $B$, and let $\tilde{u}$ be its conjugate harmonic function chosen such that $\tilde{u}(0)=0$. Since $u(0)=|B| / 2 \pi$ the holomorphic function $F=u+i \tilde{u}$ transforms $\Delta$ into the strip $S=\{\omega: 0<\operatorname{Re} \omega<1\}$. Notice that $F$ has radial boundary values except for a finite number of points, and $F$ applies the interior of $B$ into $\{\omega: \operatorname{Re} \omega=1\}$ and $\partial \Delta \backslash B$ into $\{\omega: \operatorname{Re} \omega=0\}$.

Now, let $G$ be the Riemann mapping of $S$ chosen such that

$$
G(|B| / 2 \pi)=0
$$

$G$ transforms $\{\omega: \operatorname{Re} \omega=1\}$ onto an interval $J$ of $\partial \Delta$. On the other hand, the function $h=G \circ F$ is clearly an inner function, $h(0)=0$ and $h^{-1}(I)=B$. By composing $h$ with an appropriate rotation we finish the proof of the existence statement.

To show the uniqueness of $f$, it is sufficient to prove the following

Lemma 8. If $A$ is any Borel subset of $\partial \Delta$, such that $\int_{A} e^{-i \theta} d \theta \neq 0$, and $f$, $g$ are inner functions with $f(0)=g(0)=0$ such that

$$
f^{-1}(A) \stackrel{\circ}{=} g^{-1}(A)
$$


then $f \equiv g$.

Here $\stackrel{\circ}{=}$ denotes equality up to a set of zero Lebesgue measure.

Proof. Let $F: \Delta \longrightarrow\{\omega: 0<\operatorname{Re} \omega<1\}$ be the holomorphic function given by

$$
F(z)=\frac{1}{2 \pi} \int_{A} \frac{e^{i \theta}+z}{e^{i \theta}-z} d \theta .
$$

$F$ is univalent in a neighbourhood of 0 , because

$$
F^{\prime}(0)=\frac{1}{\pi} \int_{A} e^{-i \theta} d \theta \neq 0 .
$$

Now, observe that $\operatorname{Re}(F \circ f)=\operatorname{Re}(F \circ g)$ almost everywhere on $\partial \Delta$. Since $\operatorname{Re}(F \circ f)$ and $\operatorname{Re}(F \circ g)$ are bounded harmonic functions it follows that $F \circ f=F \circ g+i c$ in $\Delta$, where $c$ is a real constant. Since $f(0)=g(0)$, we deduce that $F \circ f=F \circ g$ which proves the lemma because $F$ is univalent in a neighbourhood of 0 .

Observe that, in particular, the condition $\int_{A} e^{-i \theta} d \theta \neq 0$ is satisfied e.g. if $A$ is any interval in $\partial \Delta$ with $0<|A|<2 \pi$.

The condition $\int_{A} e^{-i \theta} d \theta \neq 0$ is not only a technicality. If $A$ is $k$-symmetrical ( i.e., there exists a subset $A_{0} \subset A$, with $A_{0} \subset[0,2 \pi / k]$, such that $A \stackrel{\circ}{=} A_{0} \cup$ $\left.\left(A_{0}+2 \pi / k\right) \cup\left(A_{0}+4 \pi / k\right) \cup \cdots \cup\left(A_{0}+2 \pi(k-1) / k\right)\right)$, and $\int_{A} e^{-i k \theta} d \theta \neq 0$, then $f=\omega g$, where $\omega$ is a $k$-th root of unity. To see this, one can use Lemma 8 with the functions $h \circ f, h \circ g$ and the set $h(A)$, where $h(z)=z^{k}$.

Also, note that if $A$ is the union of two intervals in $\partial \Delta$, then $f= \pm g$, because $\int_{A} e^{-i \theta} d \theta=0$ implies that $A$ is 2-symmetrical.

Notice that if the function $g$ in Lemma 8 were the identity, and $0<$ $|A|<2 \pi$, then, by ergodicity, we would have that $f$ is a rotation of rational angle. This, together with the above remark, could suggest that perhaps the following statement was true:

If $A$ is any Borel subset of $\partial \Delta$, such that $0<|A|<2 \pi$, and $f, g$ are inner functions with $f(0)=g(0)=0$ such that

$$
f^{-1}(A) \stackrel{\circ}{=} g^{-1}(A)
$$

then $f \equiv \lambda g$ with $|\lambda|=1$.

But this is false as the next example shows: Let $B$ be the following Blaschke product

$$
B(z)=z \frac{2 z-1}{2-z} .
$$

By applying a theorem of Stephenson [S, Theorem 3] to the pair $B,-B$, one obtains two inner functions $f$ and $g$ with $f(0)=g(0)=0$, such that

$$
B \circ f=-B \circ g \text {. }
$$


But then $(B(f))^{2}=(B(g))^{2}$, and so, if we had $f=\lambda g$, we could conclude that $B(z)=-B(\lambda z)$. But, since $B^{\prime}(0) \neq 0$, we had $\lambda=-1$, i.e., $B(z)=$ $-B(-z)$, a contradiction.

The following is well known, at least for $\alpha=0$, see for instance [A, p. 35-36] where it is credited to Beurling.

Proposition 2. Let $0 \leq \alpha<1$. If $I$ is any interval in $\partial \Delta$, then $I$ has the minimum $\alpha$-capacity between all the Borel subsets of $\partial \Delta$ with the same Lebesgue measure than $I$.

Proof. Let $E$ be a Borel set such that $|E|=|I|$. A standard approximation argument shows that for all $\varepsilon>0$ there exists a finite union $B_{\varepsilon}$ of closed intervals such that

$$
|| E|-| B_{\varepsilon}||<\varepsilon \quad \text { and } \quad\left|\operatorname{cap}_{\alpha}(E)-\operatorname{cap}_{\alpha}\left(B_{\varepsilon}\right)\right|<\varepsilon .
$$

Let $I_{\varepsilon}$ be a closed interval with the same center than $I$ and such that $\left|I_{\varepsilon}\right|=$ $\left|B_{\varepsilon}\right|$. By Lemma 7 , we can find an inner function $f_{\varepsilon}$ such that

$$
f_{\varepsilon}(0)=0 \quad \text { and } \quad f_{\varepsilon}^{-1}\left(I_{\varepsilon}\right)=B_{\varepsilon} .
$$

Therefore, by Theorem 1,

$$
\operatorname{cap}_{\alpha}(E)+\varepsilon \geq \operatorname{cap}_{\alpha}\left(B_{\varepsilon}\right) \geq \operatorname{cap}_{\alpha}\left(I_{\varepsilon}\right),
$$

but $\operatorname{cap}_{\alpha}\left(I_{\varepsilon}\right) \rightarrow \operatorname{cap}_{\alpha}(I)$ as $\varepsilon \rightarrow 0$.

The following proposition is not unexpected since ergodic theory says that $f^{-k}(E)$ is well spread on $\partial \Delta$. Hereafter $f^{k}=f \circ \cdots \circ f$ denotes the $k$-iterate of $f$ and $f^{-k}=\left(f^{k}\right)^{-1}$.

Proposition 3. If $f: \Delta \rightarrow \Delta$ is inner but not a rotation, $f(0)=0$, $0 \leq \alpha<1$ and $E$ is a Borel subset of $\partial \Delta$ with $\operatorname{cap}_{\alpha}(E)>0$, then

$$
\operatorname{cap}_{\alpha}\left(f^{-k}(E)\right) \rightarrow \operatorname{cap}_{\alpha}(\partial \Delta) \quad \text { as } k \rightarrow \infty \text {. }
$$

The proof of this result is an easy consequence of the following lemma.

Lemma 9. With the hypotheses of Proposition 3, if $\mu$ is any probability measure on $E$ with finite $\alpha$-energy and if $\nu_{k}$ is the probability measure in $f^{-k}(E)$ such that $P_{\nu_{k}}=P_{\mu} \circ f^{k}$, then

$$
I_{\alpha}\left(\nu_{k}\right) \longrightarrow I_{\alpha}\left(\frac{|\cdot|}{2 \pi}\right) \quad \text { as } k \rightarrow \infty .
$$


With this, we have

$$
\frac{1}{\operatorname{cap}_{\alpha}\left(f^{-k}(E)\right)} \leq I_{\alpha}\left(\nu_{k}\right) \longrightarrow I_{\alpha}\left(\frac{|\cdot|}{2 \pi}\right)=\frac{1}{\operatorname{cap}_{\alpha}(\partial \Delta)}
$$

giving us the conclusion of Proposition 3.

Proof of Lemma 9. We will prove it for $0<\alpha<1$; the case $\alpha=0$ being similar.

By Lemma 2 (i), we have with an appropriate function $g_{\alpha}$ that

$$
I_{\alpha}(\sigma)=\int_{0}^{1} \int_{0}^{2 \pi}\left|P_{\sigma}\left(r e^{i \theta}\right)\right|^{2} d \theta g_{\alpha}(r) d r
$$

for any probability measure $\sigma$ on $\partial \Delta$.

Using (3) we have for all $r \in(0,1)$ that

$$
\int_{0}^{2 \pi}\left|P_{\nu_{k}}\left(r e^{i \theta}\right)\right|^{2} d \theta \leq \int_{0}^{2 \pi}\left|P_{\mu}\left(r e^{i \theta}\right)\right|^{2} d \theta
$$

Since $\mu$ has finite $\alpha$-energy, the right hand side in the last inequality, as a function of $r$, belongs to $L^{1}\left(g_{\alpha}(r) d r\right)$. Therefore, by using the Lebesgue's dominated convergence theorem, we would be done if we show that

$$
\int_{0}^{2 \pi}\left|P_{\nu_{k}}\left(r e^{i \theta}\right)\right|^{2} d \theta \rightarrow \frac{1}{2 \pi} \quad \text { as } k \rightarrow \infty,
$$

for each $r$ with $0<r<1$. But, by Schwarz's lemma, and since $f$ is not a rotation, $\left|f^{k}\left(r e^{i \theta}\right)\right| \longrightarrow 0$ as $k \rightarrow \infty$, uniformly on $\theta$ for $r$ fixed. Therefore, for each $r, P_{\nu_{k}}\left(r e^{i \theta}\right)=P_{\mu}\left(f^{k}\left(r e^{i \theta}\right)\right) \longrightarrow 1 / 2 \pi$, as $k \rightarrow \infty$, uniformly on $\theta$, and this implies (9).

Even in the case when $\operatorname{cap}_{\alpha}(E)=0$, the sets $f^{-k}(E)$ are well spread on $\partial \Delta$.

Proposition 4. If $f: \Delta \longrightarrow \Delta$ is an inner function (but not a rotation) with $f(0)=0, E$ is any non empty Borel subset of $\partial \Delta$, and $\mu$ is any probability measure on $E$, then for some absolute constant $C$ and a positive constant A that only depends on $\left|f^{\prime}(0)\right|$, we have that

$$
\left|\nu_{k}(I)-\frac{|I|}{2 \pi}\right|<C e^{-A k}
$$

for each interval $I \subset \partial \Delta$. In particular,

$$
\nu_{k} \rightarrow \frac{|\cdot|}{2 \pi}
$$


in the usual weak-* topology.

Here $\nu_{k}$ is the probability measure concentrated in $f^{-k}(E)$ such that $P_{\nu_{k}}=$ $P_{\mu} \circ f^{k}$.

Proof. The proof is similar to that of Lemma 3 in $[\mathbf{P}]$, but using here the fact that $P_{\nu_{k}}=P_{\mu} \circ f^{k}$ instead of Lemma 1 in $[\mathbf{P}]$.

Proposition 5. If $f: \mathbb{B}_{n} \longrightarrow \Delta$ is inner, then $f$ assumes in $\partial \mathbb{B}_{n}$ all the values in $\partial \Delta$.

Proof. Let $f: \mathbb{B}_{n} \longrightarrow \Delta$ be an inner function. It is enough to prove that $f^{-1}\{1\} \neq \varnothing$. But,

$$
u:=\operatorname{Re}\left(\frac{1+f}{1-f}\right)=\frac{1-|f|^{2}}{|1-f|^{2}}>0, \quad \text { in } \mathbb{B}_{n} .
$$

Therefore, $u$ is harmonic and positive in $\mathbb{B}_{n}$ and so there exists a positive measure in $\mathbb{S}_{n}$ such that

$$
\operatorname{Re}\left(\frac{1+f}{1-f}\right)=P_{\mu} .
$$

By (10) $P_{\mu}$ tends radially to 0 a.e. with respect to Lebesgue measure, since $f$ is inner and (by Privalov's theorem, (see e.g., [R, Theorem 5.5.9])) $f$ can assume the value 1 at most in a set of zero Lebesgue measure. Then, the Radon-Nikodym derivative of $\mu$ with respect to Lebesgue measure is zero a.e., and so $\mu$ is a singular measure.

By Lemma 11 it follows that $P_{\mu} \rightarrow+\infty$ in a set of full $\mu$-measure. But this is the same to say that $f\left(r e^{i \theta}\right) \rightarrow 1$ in that set.

When the inner function $f$ has order $k \geq 1$ at 0 , we can improve Theorem 1 in the case $\alpha=0$.

Theorem 3. If $f: \Delta \longrightarrow \Delta$ is inner,

$$
f(0)=f^{\prime}(0)=\cdots=f^{(k-1)}(0)=0, \quad f^{(k)}(0) \neq 0, \quad(k \geq 1),
$$

and $E$ is a Borel subset of $\partial \Delta$, then

$$
\operatorname{cap}_{0}\left(f^{-1}(E)\right) \geq\left(\operatorname{cap}_{0}(E)\right)^{1 / k} .
$$

Moreover, if $\operatorname{cap}_{0}(E)>0$, equality holds if and only if either $f(z)=\lambda z^{k}$, with $|\lambda|=1$, or $\operatorname{cap}_{0}(E)=\operatorname{cap}_{0}(\partial \Delta)$.

Proof. For such a function $f$, Schwarz's lemma says us that $|f(z)| \leq|z|^{k}$, with equality only if $f(z)=\lambda z^{k}$ with $|\lambda|=1$. With this in mind, the 
subordination principle says now (see e.g. $[\mathbf{H H}]$ ) that if $v$ is a subharmonic function in $\Delta$, then

$$
\int_{0}^{2 \pi} v\left(f\left(r e^{i \theta}\right)\right) d \theta \leq \int_{0}^{2 \pi} v\left(r^{k} e^{i \theta}\right) d \theta
$$

with equality for a given $r$ only if $v$ is harmonic in $\{|z|<r\}$ or $f$ is a rotation of $z^{k}$.

Now, in order to prove (11), we can assume that $E$ is closed. If $\mu_{e}$ is the equilibrium probability distribution of $E$ and $\nu$ is the probability measure in $f^{-1}(E)$ such that $P_{\nu}=P_{\mu} \circ f$, then

$$
\begin{aligned}
I_{0}(\nu) & =2 \pi \int_{0}^{1} \int_{0}^{2 \pi}\left|P_{\mu_{e}}\left(f\left(r e^{i \theta}\right)\right)-\frac{1}{2 \pi}\right|^{2} d \theta \frac{d r}{r} \\
& \leq 2 \pi \int_{0}^{1} \int_{0}^{2 \pi}\left|P_{\mu_{e}}\left(r^{k} e^{i \theta}\right)-\frac{1}{2 \pi}\right|^{2} d \theta \frac{d r}{r} .
\end{aligned}
$$

Substituting $r^{k}=t$, we obtain that

$$
I_{0}(\nu) \leq \frac{1}{k} I_{0}\left(\mu_{e}\right) .
$$

This finishes the proof of (11). The equality statement can be proved in the same way as that of Theorem 1 .

Remark. For other $\alpha$ 's $(0<\alpha<1)$ we can show

$$
\frac{1}{\operatorname{cap}_{\alpha}\left(f^{-1}(E)\right)}-\frac{1}{\operatorname{cap}_{\alpha}(\partial \Delta)} \leq \frac{C_{\alpha}}{k^{1-\alpha}}\left(\frac{1}{\operatorname{cap}_{\alpha}(E)}-\frac{1}{\operatorname{cap}_{\alpha}(\partial \Delta)}\right)
$$

where $C_{\alpha}$ is a constant depending only on $\alpha$.

We expect $C_{\alpha}=1$, but we have not been able to show this.

\section{Distortion of $\alpha$-content.}

The following is an extension of Löwner's lemma.

Theorem 4. If $f: \mathbb{B}_{n} \longrightarrow \Delta$ is inner, $f(0)=0$ and $E$ is a Borel subset of $\partial \Delta$, then, for $0<\alpha \leq 1$,

$$
M_{2 n-2+\alpha}\left(f^{-1}(E)\right) \geq C_{n, \alpha} M_{\alpha}(E)
$$

and

$$
\mathcal{M}_{2(n-1+\alpha)}\left(f^{-1}(E)\right) \geq C_{n, \alpha}^{\prime} M_{\alpha}(E) .
$$


Here $M_{\beta}$ and $\mathcal{M}_{\beta}$ denote, respectively, $\beta$-dimensional content with respect to the euclidean metric and with respect to the metric in $\mathbb{S}_{n}$ given by

$$
d(a, b)=|1-\langle a, b\rangle|^{1 / 2}
$$

where $\langle a, b\rangle=\sum a_{j} \bar{b}_{j}$ is the inner product in $\mathbb{C}^{n}$. This metric is equivalent to the Carnot-Carathèodory metric in the Heisenberg group model for $\mathbb{S}_{n}$. We refer to $[\mathbf{R}]$ for details about this metric.

Recall that in a general metric space $(X, d)$ the $\alpha$-content of a set $E \subset X$ is defined as

$$
M_{\alpha}(E)=\inf \left\{\sum_{i} r_{i}^{\alpha}: E \subset \bigcup_{i} B_{d}\left(x_{i}, r_{i}\right)\right\} .
$$

Observe that, as a consequence of Theorem 4, one obtains

Corollary. If $f: \mathbb{B}_{n} \longrightarrow \Delta$ is inner and $E$ is a Borel subset of $\partial \Delta$, then

$$
\operatorname{Dim}\left(f^{-1}(E)\right) \geq 2 n-2+\operatorname{Dim}(E)
$$

and

$$
\operatorname{Dim}\left(f^{-1}(E)\right) \geq 2 n-2+2 \operatorname{Dim}(E)
$$

where Dim and Dim denote, respectively, Hausdorff dimension with respect to the euclidean metric and the metric d.

In order to prove Theorem 4 we will prove a lemma about Poisson integrals. We need to consider the classical Poisson kernel (not normalized)

$$
P(\xi, z)=\frac{1-|z|^{2}}{|\xi-z|^{2 n}} \quad\left(z \in \mathbb{B}_{n}, \xi \in \mathbb{S}_{n}\right)
$$

and the invariant Poisson kernel

$$
Q(\xi, z)=\frac{\left(1-|z|^{2}\right)^{n}}{|1-\langle z, \xi\rangle|^{2 n}} \quad\left(z \in \mathbb{B}_{n}, \xi \in \mathbb{S}_{n}\right)
$$

Of course, they coincide if $n=1$. In this section if $\nu$ is a positive measure in $\mathbb{S}_{n}$, we will denote by $P_{\nu}$ the function

$$
P_{\nu}(z)=\int_{\mathbb{S}_{n}} P(\xi, z) d \nu(\xi)
$$

and by $Q_{\nu}$ the invariant Poisson extension of $\nu$

$$
Q_{\nu}(z)=\int_{\mathbb{S}_{n}} Q(\xi, z) d \nu(\xi)
$$


Lemma 10. Let $\mu$ be a finite positive measure in $\partial \Delta$, and let $f: \mathbb{B}_{n} \rightarrow \Delta$ be an inner function. Then, there exists a finite measure $\nu \geq 0$ in $\mathbb{S}_{n}$ such that $P_{\mu} \circ f=P_{\nu}$, and if $\nu$ has singular part $\sigma$ and continuous part $\gamma$, and we denote by $A$ the set

$$
A=\left\{\xi \in \mathbb{S}_{n}: P_{\sigma}(r \xi) \rightarrow+\infty, \text { as } r \rightarrow 1\right\}
$$

and by $B$ the set

$$
B=\left\{\xi \in \mathbb{S}_{n}: \exists \lim _{r \rightarrow 1} f(r \xi)=f(\xi),|f(\xi)|=1 \text { and } \lim _{r \rightarrow 1} P_{\gamma}(r \xi)>0\right\},
$$

then $A$ has full $\sigma$-measure, $B$ has full $\gamma$-measure and

$$
A \cup B \subset f^{-1}(\operatorname{support} \mu)
$$

and so

$$
\nu\left(f^{-1}(\operatorname{support} \mu)\right)=\|\nu\| .
$$

The same is true if we replace $P_{\nu}$ by $Q_{\nu^{\prime}}\left(P_{\mu} \circ f=Q_{\nu^{\prime}}\right)$ and $A, B$ by the following sets

$$
A^{\prime}=\left\{\xi \in \mathbb{S}_{n}: Q_{\sigma^{\prime}}(r \xi) \rightarrow+\infty, \text { as } r \rightarrow 1\right\}
$$

and

$$
B^{\prime}=\left\{\xi \in \mathbb{S}_{n}: \exists \lim _{r \rightarrow 1} f(r \xi)=f(\xi),|f(\xi)|=1 \text { and } \lim _{r \rightarrow 1} Q_{\gamma^{\prime}}(r \xi)>0\right\}
$$

where $\sigma^{\prime}$ and $\gamma^{\prime}$ denote, respectively, the singular and the continuous part of $\nu^{\prime}$.

Proof. We will prove the lemma only for the measure $\nu^{\prime}$, since the proof of the result for $\nu$ is similar and standard.

Let $U: \Delta \longrightarrow \mathbb{C}$ be a holomorphic function such that $\operatorname{Re} U=P_{\mu}$. Then $U \circ f$ is also holomorphic and so $\operatorname{Re}(U \circ f)=P_{\mu} \circ f$ is pluriharmonic, i.e. harmonic and $\mathcal{M}$-harmonic (see e.g. [R, Theorem 4.4.9]). Therefore there exist finite positive measures $\nu$ and $\nu^{\prime}$ in $\mathbb{S}_{n}$ such that

$$
P_{\mu} \circ f=P_{\nu}, \quad P_{\mu} \circ f=Q_{\nu^{\prime}} .
$$

Let us denote by $E$ the support of $\mu$. If $\xi \in A^{\prime}$, then $|f(r \xi)| \rightarrow 1$ as $r \rightarrow 1$. The curve $\{f(r \xi): 0 \leq r<1\}$ in $\Delta$ must end on a unique point $e^{i \psi}=f(\xi) \in \Delta$, since otherwise we would have $P_{\mu} \equiv+\infty$ on a set of positive Lebesgue measure. Now, $e^{i \psi} \in E$, since otherwise $P_{\mu}$ vanishes continuously at $e^{i \psi}$. Therefore $A^{\prime} \subset f^{-1}(E)$. Similarly one sees that $B^{\prime} \subset f^{-1}(E)$. 
The set $A^{\prime}$ has full $\sigma^{\prime}$-measure since by the inequality (14), that we will prove later,

$$
\left\{\xi \in \mathbb{S}_{n}: \underset{-}{\mathrm{D}} \sigma^{\prime}(\xi)=\infty\right\} \subset A^{\prime}
$$

where

$$
\underline{\mathrm{D}} \sigma^{\prime}(\xi)=\liminf _{r \rightarrow 0} \frac{\sigma^{\prime}\left(B_{d}(\xi, r)\right)}{\left|B_{d}(\xi, r)\right|},
$$

and the set $\left\{\xi: \underline{\mathrm{D}} \sigma^{\prime}(\xi)=\infty\right\}$ has full $\sigma^{\prime}$-measure (see Lemma 11 below). Let us observe that $([\mathbf{R}$, p. 67])

$$
\left|B_{d}(\xi, r)\right| \sim r^{2 n}
$$

The set $B^{\prime}$ has full $\gamma^{\prime}$-measure, since as $r \rightarrow 1$

$$
Q_{\gamma^{\prime}}(r \xi) \longrightarrow \frac{d \gamma^{\prime}}{d L} \quad \text { a.e. }
$$

with respect to Lebesgue measure $L$ (see, e.g., $[\mathbf{R}$, Theorem 5.4.9]) and $\left\{\frac{d \gamma^{\prime}}{d L}>0\right\}$ has full $\gamma^{\prime}$-measure.

Lemma 11. Suppose that $\mu$ is a singular positive Borel measure (with respect to Lebesgue measure) in $\mathbb{S}_{n}$. Then

$$
\underline{\mathrm{D}} \mu(x)=\infty \quad \text { a.e. } \mu .
$$

Proof. Let $\mathcal{A}$ be a Borel set such that $|\mathcal{A}|=0$, and $\mu$ is concentrated on $\mathcal{A}$. Define for $\alpha>0$

$$
\mathcal{A}_{\alpha}=\{x \in \mathcal{A}: \underline{\mathrm{D}} \mu(x)<\alpha\} .
$$

It is enough to prove that $\mu\left(\mathcal{A}_{\alpha}\right)=0$, and by regularity that $\mu(K)=0$ for all $K$ compact subset of $\mathcal{A}_{\alpha}$.

Fix $\varepsilon>0$. Since $K \subset \mathcal{A}_{\alpha} \subset \mathcal{A},|K|=0$ and so there exists an open set $V$ with $K \subset V$ and $|V|<\varepsilon(|\cdot|$ denotes Lebesgue measure).

Now, for each $x \in K$, we can find $r_{x}>0$ such that

$$
\frac{\mu\left(B_{d}\left(x, r_{x}\right)\right)}{\left|B_{d}\left(x, r_{x}\right)\right|}<\alpha \quad \text { and } \quad B_{d}\left(x, r_{x} / 3\right) \subset V .
$$

The family $\left\{B_{d}\left(x, r_{x} / 3\right): x \in K\right\}$ covers $K$, hence we can extract a finite subcollection $\Phi$ that also covers $K$. Now, using a Vitaly-type lemma (see, e.g., [R, Lemma 5.2.3]), we can find a disjoint subcollection $\Gamma$ of $\Phi$ such that

$$
K \subset \bigcup_{\Gamma} B_{d}\left(x_{\imath}, r_{x_{i}}\right)
$$


Note that as a consequence of Proposition 5.1.4 in $[\mathbf{R}]$ we have that

$$
\Theta_{d}:=\sup _{\delta} \frac{\left|B_{d}\left(x, r_{x}\right)\right|}{\left|B_{d}\left(x, r_{x} / 3\right)\right|}<\infty
$$

Therefore

$$
\begin{aligned}
\mu(K) & \leq \sum_{\Gamma} \mu\left(B_{d}\left(x_{i}, r_{x_{i}}\right)\right)<\alpha \sum_{\Gamma}\left|B_{d}\left(x_{i}, r_{x_{i}}\right)\right| \\
& <\Theta_{d} \alpha \sum_{\Gamma}\left|B_{d}\left(x_{i}, r_{x_{i}} / 3\right)\right| \leq \Theta_{d} \alpha|V|<\Theta_{d} \alpha \varepsilon .
\end{aligned}
$$

Proof of Theorem 4. We will prove only (ii), since (i) is obtained in a similar way.

Assume, as we may, that $E$ is a closed subset of $\partial \Delta$ and $M_{\alpha}(E)>0$. Then, see e.g. [T, p. 64], there exists a positive mass distribution on $E$ of finite total mass, such that: (a) $\mu(E)=M_{\alpha}(E)$, (b) $\mu(I) \leq C_{\alpha}|I|^{\alpha}$ for any open interval $I$, where $C_{\alpha}$ is a constant independent of $E$. A standard estimate shows that

$$
P_{\mu}(z) \leq \frac{C_{\alpha}}{(1-|z|)^{1-\alpha}}, \quad(z \in \Delta)
$$

with $C_{\alpha}$ a new constant. Let $\nu^{\prime} \geq 0$ be a measure in $\mathbb{S}_{n}$ such that $P_{\mu} \circ f=$ $Q_{\nu^{\prime}}$. Schwarz's lemma (see e.g. $[\mathbf{R}$, Theorem 8.1.2]) and (12) give the corresponding inequality for $\nu^{\prime}$ :

$$
Q_{\nu^{\prime}}(z) \leq \frac{C_{\alpha}}{(1-\|z\|)^{1-\alpha}}, \quad\left(z \in \mathbb{B}_{n}\right) .
$$

We claim that for each $z \in \mathbb{B}_{n}$

$$
Q_{\nu^{\prime}}(z) \geq C_{n} \frac{\nu^{\prime}\left(B_{d}\left(\xi,(2(1-\|z\|))^{1 / 2}\right)\right)}{(1-\|z\|)^{n}}, \quad\left(z \in \mathbb{B}_{n}\right)
$$

where $\xi=z /\|z\|$ and $B_{d}(\xi, R)$ denotes the $d$-ball with center $\xi$ and radius $R$.

Assuming (14) for the moment and using (13), we obtain that

$$
\nu^{\prime}\left(B_{d}(\xi, R)\right) \leq C_{n, \alpha} R^{2(n-1+\alpha)}, \quad\left(\xi \in \mathbb{S}_{n}, R>0\right) .
$$

If we cover the set $A^{\prime} \cup B^{\prime}$ (see Lemma 14) with $d$-balls of radii $R_{i}$, we see by (15) that

$$
\nu^{\prime}\left(A^{\prime} \cup B^{\prime}\right) \leq C_{n, \alpha} \sum_{i} R_{i}^{2(n-1+\alpha)}
$$


and so

$$
\begin{aligned}
\left\|\nu^{\prime}\right\|=\nu^{\prime}\left(A^{\prime} \cup B^{\prime}\right) & \leq C_{n, \alpha} \mathcal{M}_{2(n-1+\alpha)}\left(A^{\prime} \cup B^{\prime}\right) \\
& \leq C_{n, \alpha} \mathcal{M}_{2(n-1+\alpha)}\left(f^{-1}(E)\right) .
\end{aligned}
$$

So, since $f(0)=0$,

$$
M_{\alpha}(E)=\|\mu\|=\left\|\nu^{\prime}\right\| \leq C_{n, \alpha} \mathcal{M}_{2(n-1+\alpha)}\left(f^{-1}(E)\right) .
$$

Therefore, in order to finish the proof, it remains only to prove (14). Observe first that we can assume that $\xi=e_{1}=(1,0, \ldots, 0)$ since $d$ is invariant under the unitary transformations of $\mathbb{S}_{n}$ for the inner product $\langle\cdot, \cdot\rangle$. Now, if $z=r e_{1}$, write $\delta^{2}=2(1-r)$. If $\eta \in B_{d}\left(e_{1}, \delta\right)$, then

$$
\left|1-r \eta_{1}\right| \leq\left|1-\eta_{1}\right|+\left|\eta_{1}\right|(1-r) \leq 3(1-r) .
$$

Hence, if $\eta \in B_{d}\left(e_{1}, \delta\right)$

$$
Q(\eta, z)=\left(\frac{1-r^{2}}{\left|1-r \eta_{1}\right|^{2}}\right)^{n} \geq \frac{9^{-n}}{(1-r)^{n}}
$$

Since $Q$ is invariant under the action of the unitary group for the inner product $\langle\cdot, \cdot\rangle$ in $\mathbb{S}_{n}$, we obtain that if $z=r \xi$ and $\eta \in B_{d}(\xi, \delta)$, then

$$
Q(\eta, z) \geq \frac{9^{-n}}{(1-r)^{n}}
$$

Finally,

$$
Q_{\nu^{\prime}}(z) \geq \int_{B_{d}(\xi, \delta)} Q(\eta, z) d \nu^{\prime}(\eta) \geq 9^{-n} \frac{\nu^{\prime}\left(B_{d}(\xi, \delta)\right)}{(1-r)^{n}}
$$

\section{Distortion of subsets of the disc.}

We have discussed how inner functions distort boundary sets. There are some results on how they distort subsets of $\Delta$. On the one hand Hamilton $[\mathbf{H}]$ has shown that

Theorem H. For all Borel subsets $E$ of $\Delta$,

$$
H_{\alpha}\left(f^{-1}(E)\right) \geq H_{\alpha}(E), \quad 0<\alpha \leq 1,
$$


where $H_{\alpha}$ denotes $\alpha$-Hausdorff measure.

One naturally expects the following to be true:

If $f: \Delta \longrightarrow \Delta$ is inner, $f(0)=0$ and $E$ is a Borel subset of $\Delta$, then

$$
\operatorname{cap}_{\alpha}\left(f^{-1}(E)\right) \geq \operatorname{cap}_{\alpha}(E) .
$$

This we can prove only if $\alpha=0$. The idea comes from [P1, p. 336].

Theorem 5. Let $f: \Delta \longrightarrow \Delta$ be an inner function. If for some $k \geq 1$

$$
f(0)=f^{\prime}(0)=\cdots=f^{(k-1)}(0)=0, \quad f^{(k)}(0) \neq 0,
$$

then,

$$
\operatorname{cap}_{0}\left(f^{-1}(E)\right) \geq\left(\operatorname{cap}_{0}(E)\right)^{1 / k},
$$

for all Borel subsets of $\Delta$. Moreover, this inequality is sharp.

Sketch of proof. By approximation, it is enough to prove it if $E$ is closed and $f$ is a finite Blaschke product. Let $f$ be

$$
f(z)=z^{k} \prod_{j=1}^{d} e^{i \nu_{\jmath}} \frac{z-a_{j}}{1-\bar{a}_{\jmath} z} .
$$

Denote by $g_{E}, g_{F}$ the Green's functions of the unbounded connected component of $\hat{\mathbb{C}} \backslash E$ and $\hat{\mathbb{C}} \backslash F$ (here $F=f^{-1}(E)$ ) with pole at $\infty$. Therefore,

$$
\begin{aligned}
& g_{E}(z)-\log |z|=\log \frac{1}{\operatorname{cap}_{0}(E)}+O\left(|z|^{-1}\right), \\
& g_{F}(z)-\log |z|=\log \frac{1}{\operatorname{cap}_{0}(F)}+O\left(|z|^{-1}\right),
\end{aligned}
$$

as $|z| \rightarrow \infty$. Moreover, since $k \geq 1$

$$
g_{E}(f(z))-k \log |z|+\log \prod_{j=1}^{d}\left|a_{j}\right|=\log \frac{1}{\operatorname{cap}_{0}(E)}+O\left(|z|^{-1}\right)
$$

as $|z| \rightarrow \infty$. It is easy to see that

$$
g_{E}(f(z))-\sum_{j=1}^{d} g_{F}\left(z, \bar{a}_{j}^{-1}\right)
$$

is harmonic in the unbounded connected component of $\mathbb{C} \backslash\left(F \cup\left(\cup_{j=1}^{d}\left\{\bar{a}_{j}^{-1}\right\}\right)\right)$ and it is bounded at the points $\bar{a}_{j}^{-1}$ (here $g_{F}\left(z, \bar{a}_{\jmath}^{-1}\right)$ denotes the Green's 
function of the unbounded connected component of $\hat{\mathbb{C}} \backslash F$ with pole at $\left.\bar{a}_{j}^{-1}\right)$. Therefore, the function

$$
G(z)=\frac{1}{k} g_{E}(f(z))-g_{F}(z)-\frac{1}{k} \sum_{j=1}^{d} g_{F}\left(z, \bar{a}_{j}^{-1}\right)
$$

is harmonic and bounded in the unbounded connected component of $\hat{\mathbb{C}} \backslash F$. Since $G=0$ on the outer boundary of $F$, it follows that $G \equiv 0$.

Now, by using the symmetry of Green's function, we have that

$$
g_{F}\left(z, \bar{a}_{j}^{-1}\right) \longrightarrow g_{F}\left(\bar{a}_{j}^{-1}\right), \quad \text { as }|z| \rightarrow \infty,
$$

and so, from (16),

$$
\log \frac{1}{\operatorname{cap}_{0}(E)}-\log \prod_{j=1}^{d}\left|a_{j}\right|-k \log \frac{1}{\operatorname{cap}_{0}(F)}-\sum_{j=1}^{d} g_{F}\left(\bar{a}_{j}^{-1}\right)=0 .
$$

On the other hand, since $F \subset \Delta$, the maximum principle says that

$$
g_{F}(z) \geq g_{\Delta}(z)=\log |z|, \quad|z|>1 .
$$

Hence, from (17), we obtain that

$$
\log \frac{1}{\operatorname{cap}_{0}(E)}-\log \prod_{j=1}^{d}\left|a_{\jmath}\right|-k \log \frac{1}{\operatorname{cap}_{0}(F)} \geq \sum_{j=1}^{d} \log \left|a_{j}\right|^{-1},
$$

and the inequality in the theorem follows.

Finally, to show that the inequality is sharp one simply has to consider the function $f(z)=z^{k}$.

\section{References}

[A] L.V. Ahlfors, Conformal invariants, McGraw-Hill, 1973.

[AS] M. Abramowitz and I.A. Stegun, Handbook of Mathematical functions, Dover Pub. Inc., nine-th printing, 1970.

[B] A. Beurling, Ensembles exceptionnels, Acta Math., 72 (1939), 1-13.

[C] L. Carleson, Selected problems on exceptional sets, Van Nostrand, 1967.

[FP] J.L. Fernández and D. Pestana, Distortion of boundary sets under inner functions and applications, Indiana Univ. Math. J., 41 (1992), 439-447.

[H] D. Hamilton, Distortion of sets by inner functions, Proc. Amer. Math. Soc., 117 (1993), 771-774.

[HH] R.R. Hall and W.K. Hayman, A problem in the theory of subordination, J. d'Analyse, 60 (1993), 99-111. 
[KS] J.P. Kahane and R. Salem, Ensembles parfaits et Sèries Trigonomètriques, Hermann, 1963.

[L] N.S. Landkof, Foundations of modern potential theory, Springer-Verlag, 1972.

[N] J.H. Neuwirth, Ergodicity of some mappings of the circle and the line, Israel J. Math., 31 (1978), 359-367.

[P] Ch. Pommerenke, Ergodic properties of inner functions, Math. Ann., 256 (1981), 43-50.

[P1] Univalent functions, Vandenhoek und Ruprecht, Göttingen, 1975.

[R] W. Rudin, Function theory in the unit ball of $\mathbb{C}^{n}$, Springer-Verlag, 1980.

[R1] - New constructions of functions holomorphic in the unit ball of $\mathbb{C}^{n}$, Lecture presented at the NSF-CBMS Regional Conference hosted by Michigan State University (1985).

[S] K. Stephenson, Analytic functions and hypergroups of function pairs, Indiana Univ. Math. J., 31 (1982), 843-884.

[SW] E. Stein and G. Weiss, Introduction to Fourier Analysis on euclidean spaces, Princeton University Press, 1971.

[T] M. Tsuji, Potential theory in Modern Function theory, Chelsea, 1959.

Received July 29, 1993 and revised September 12, 1994.

Universidad Autónoma de Madrid

28049 MADRID, SPAIN

E-mail address: pando@ccuam3.sdi.uam.es

Universidad Autónoma de Madrid

28049 MADRID, SPAIN

E-mail address: madom@ccuam3.sdi.uam.es

AND

Universidad Carlos III de Madrid

Butarque, 15

LEGANÉS, 28911 MADRID, SPAIN 




\section{PACIFIC JOURNAL OF MATHEMATICS}

Volume $172 \quad$ No. $1 \quad$ January 1996

A class of incomplete non-positively curved manifolds

BRIAN BOWDITCH

The quasi-linearity problem for $C^{*}$-algebras

41

L. J. BUNCE and JOHN DAVID MAITLAND WRIGHT

Distortion of boundary sets under inner functions. II

Jose Luis Fernandez Perez, Domingo Pestana and José Rodríguez

Irreducible non-dense $A_{1}^{(1)}$-modules

VJACHESLAV M. FUTORNY

$M$-hyperbolic real subsets of complex spaces

101

Giuliana Gigante, Giuseppe Tomassini and Sergio Venturini

Values of Bernoulli polynomials

ANDREW GRANVILLE and ZHI-WEI SUN

The uniqueness of compact cores for 3-manifolds

LUKE HARRIS and PETER SCOTT

Estimation of the number of periodic orbits

BOJU JIANG

Factorization of $p$-completely bounded multilinear maps

Christian Le MERdy

Finitely generated cohomology Hopf algebras and torsion

JAMEs Peicheng LiN

The positive-dimensional fibres of the Prym map

JUAN-CARLOS NARANJO

Entropy of a skew product with a $Z^{2}$-action

KYEWON KOH PARK

Commuting co-commuting squares and finite-dimensional Kac algebras

TAKASHI SANO

Second order ordinary differential equations with fully nonlinear two-point boundary 255 conditions. I

H. BEVAN THOMPSON

Second order ordinary differential equations with fully nonlinear two-point boundary conditions. II

H. BEVAN THOMPSON

The flat part of non-flat orbifolds

FENG XU 